\title{
Differences in the perceived impact of sleep deprivation among surgical and non-surgical residents
}

Sarah I Woodrow, ${ }^{1,2}$ Jason Park, ${ }^{3}$ Brian J Murray, ${ }^{4}$ Calvin Wang, ${ }^{2}$ Mark Bernstein, ${ }^{1}$ Richard K Reznick ${ }^{1,2}$ \& Stanley J Hamstra ${ }^{5}$

OBJECTIVE Resident work hour restrictions have been mandated in the USA largely out of concern that sleep deprivation compromises doctor performance and patient care. However, individuals' ability to recognise the effects of sleep deprivation has not been studied in medical education. We examined the perceived impact of sleep deprivation among different groups of postgraduate medical trainees.

METHODS A survey addressing work hours, sleepiness and daily functioning was mailed to all residents in the internal medicine, surgery and psychiatry programmes at the University of Toronto who were working at 6 different teaching hospitals. The mailing included the Epworth Sleepiness Scale (ESS), measuring acute sleepiness, and a new Sleep Deprivation Impact (SDI) scale, consisting of 12 items designed to measure the perceived impact of sleep deprivation on an individual's own performance.

RESULTS Overall, $62.5 \%$ of surgery $(95 / 152)$ and $59.5 \%$ of non-surgery residents (194/326) completed the survey. Surgery residents reported working longer hours per week (83.0 versus 62.5 hours; $P<0.01$ ), and scored higher on the ESS (12.8 versus 9.2; $P<0.01)$ compared with other residents. Surgery

\footnotetext{
${ }^{1}$ Department of Surgery, University of Toronto, Toronto, Ontario, Canada

${ }^{2}$ Wilson Centre for Research in Education, University of Toronto, Toronto, Ontario, Canada

${ }^{3}$ Department of Surgery, University of Manitoba, Winnipeg, Manitoba, Canada

${ }^{4}$ Division of Neurology, Department of Medicine, Sunnybrook and Women's College Health Sciences Centre, Toronto, Ontario, Canada

${ }^{5}$ Department of Medical Education, University of Michigan Medical School, Ann Arbor, Michigan, USA
}

Correspondence: Stanley J Hamstra, Department of Medical Education, G1208 Towsley, SPC 5201, 1500 East Medical Center Drive, Ann Arbor, Michigan, 48109-5201, USA.

Tel: 001734763 1424; Fax: 001734936 1641;

E-mail: shamstra@umich.edu residents scored significantly lower than others on the SDI scale ( 45.2 versus $51.5, P<0.01$ ), indicating less perceived impact of sleep deprivation on performance.

CONCLUSIONS These results are consistent with the presence of an underlying culture within surgery in which individuals may be less willing to accept a natural limitation of individual performance. Whether these findings represent an actual resilience to sleep deprivation among surgery residents or a misperception within this group remains to be determined.

KEYWORDS humans; male; female; adult; sleep deprivation/ *psychology; *internship and residency; *perception; *attitude of health personnel; surgery/*education; clinical competence/ *standards.

Medical Education 2008: 42: 459-467 doi:10.1111/j.1365-2923.2007.02963.x

\section{INTRODUCTION}

Long hours and little sleep have long been considered a rite of passage in medical training. However, pressure from within the medical community and society at large have recently resulted in changes to this practice in the interest of patient safety. These changes are consistent with overwhelming evidence from laboratory-based sleep studies that suggest sleep deprivation impairs mood, cognitive and motor performance. ${ }^{1}$ Likewise, recent studies of doctors have demonstrated that they do not perform as effectively after a night without sleep ${ }^{2}$ and as a result may be at risk for making increased medical errors. ${ }^{3}$ A recent study by Arnedt $e t a l$. investigating acute sleep deprivation demonstrated impairments in neurobehavioural functioning following a night on-call that was equivalent to functioning in an acutely intoxicated state. ${ }^{4}$ Chronic partial sleep deprivation can also be significant. Although there are slight 


\section{Overview}

\section{What is already known on this subject}

Sleep deprivation affects performance on a variety of general tasks. However, some people believe that sleep deprivation does not affect them, in the face of evidence to the contrary.

\section{What this study adds}

On a day-to-day basis, surgery residents report a higher level of subjective sleepiness than other groups of residents, but surgery trainees believe that sleep deprivation does not affect them, compared with trainees in psychiatry or internal medicine.

\section{Suggestions for further research}

Further research should test surgery trainees directly for cognitive or technical performance while under conditions of sleep deprivation to determine if the perceived effects of sleep deprivation are accurate. practising doctors and compliance with work hour regulations among doctor trainees are ultimately selfregulated, denial of the effects of fatigue, like any possible source of impairment, may lead tired practitioners to continue working in a compromised state.

There is also evidence that different medical specialties may not hold the same perceptions of the effects of sleep deprivation on performance. One survey demonstrated that a higher proportion of surgeons $(70 \%)$ denied the effects of fatigue on their clinical performance, compared with anaesthetists $(47 \%){ }^{13}$

The current study examined the work patterns of residents at a large, urban academic institution and the perceived impact of sleep deprivation on their individual performances. In particular, we were interested in determining if any differences in perception exist between different groups of resident trainees. Our hypothesis was that surgery residents would report more resilience to the effects of sleep deprivation, as a reflection of the underlying culture of surgery.

\section{METHODS}

\section{Survey design}

The survey instrument was developed from a review of the literature in consultation with a diplomate of the American Board of Sleep Medicine (author BJM). Resident doctors from different specialties were also consulted to ensure applicability and comprehensiveness of the issues being studied. The survey was designed to elicit several types of information, including basic demographic data, current work hour practices and sleep requirements. Eighteen questions were formatted on a 5-point Likert scale to examine perceptions about the impact of sleep deprivation on clinical performance, including effects on professional behaviour and patient care. Of these 18 items, 12 were identified as relevant to the entire study population. Questions addressing technical or surgical skills, for example, were omitted from the scale as they would not apply to psychiatry residents. The scores for these 12 questions were summed and the resulting index referred to as the Sleep Deprivation Impact (SDI) scale. Higher scores reflect greater concern about perceived problems caused by sleep loss.

Also included in the survey were questions regarding individual ability to adapt to a sleep-deprived state, 
post-call work hours and a modified version of the Epworth Sleepiness Scale (ESS). ${ }^{14}$ The ESS requires individuals to rate their immediate likelihood of falling asleep in a number of common scenarios. It is used to distinguish between highly alert and sleepy individuals and has been validated against objective measures of sleepiness. ${ }^{15}$ In this study the ESS was modified slightly such that participants were asked to reflect back on a typical time when on rotation in their own specialty and not post-call. Prior to distribution, the survey instrument was piloted in a small group $(n=6)$ of junior residents for acceptability and readability of items.

\section{Subjects}

Paper surveys were mailed to all surgery $(n=152)$, internal medicine $(n=221)$ and psychiatry $(n=102)$ residents at the University of Toronto in postgraduate year 2 (PGY-2) and above. These specialties were chosen to reflect a maximal diversity of trainees with respect to work patterns and attitudes. Residents at this institution undertake their clinical training by rotating through 6 large public hospitals. A total of 3 mailings occurred. Separate response cards allowed for tracking of respondents in order to minimise repeated mailings to individuals who had completed the survey, while ensuring anonymity of respondents.

\section{Data analysis}

All statistical analyses were performed using a commercially available computer software package (sPSS Version 11.5; SPSS Inc., Chicago, IL, USA). Standard descriptive statistics (frequencies, percentages and means) were used to characterise the sample population. Student's $t$-tests and chi-square analyses were performed to look for group differences based on specialty, gender, marital status and level of training. Cronbach's alpha was calculated to measure the internal consistency of the 12-item SDI score. The proportion of the total variance in SDI score that is attributed to group (eta-square) was also determined.

\section{RESULTS}

\section{Demographics, work hours and sleepiness}

Overall, $62.5 \%(95 / 152)$ of surgery and $59.5 \%$ (194/326) of non-surgery residents completed the survey. Surgery residents reported working longer hours per week (83.0 versus 62.5 hours; $P<0.01$ ) compared with other residents and scored significantly higher on the ESS, indicating they felt more sleepy than non-surgery residents (12.8 versus 9.2; $P<0.01$ ) (Table 1).

Table 1 Participant demographics and work hours

\begin{tabular}{|c|c|c|}
\hline & $\begin{array}{l}\text { Surgery residents } \\
(n=95)\end{array}$ & $\begin{array}{l}\text { Non-surgery residents } \\
(n=194)\end{array}$ \\
\hline Mean age (range), years & $30.5(24-43)$ & $30.1(23-49)$ \\
\hline Females, $n(\%)$ & $15(15.8)^{\dagger}$ & $91(46.9)$ \\
\hline No. single, $n(\%)$ & $23(24.2)^{\dagger}$ & $71(36.6)$ \\
\hline No. with children, $n$ (\%) & $28(29.5)$ & $36(18.6)$ \\
\hline PGY-2, $n(\%)$ & $17(17.9)$ & $51(26.3)$ \\
\hline PGY-3 or $4, n(\%)$ & $44(46.3)$ & $96(49.5)$ \\
\hline PGY-5 and above, $n(\%)$ & $33(34.7)$ & $47(24.2)$ \\
\hline No. self-reported hours worked per week (on-service) (SD) & $83.0(12.1)^{*}$ & $62.5(16.1)$ \\
\hline Epworth Sleepiness Scale score (SD) & $12.84(4.7)^{\star}$ & $9.17(4.8)$ \\
\hline Typical time to go home post-call (SD) & $17: 11(2: 05)^{\star}$ & 13:04 (1:52) \\
\hline Hours of sleep on a typical night on call (SD) & $3.26(1.36)$ & $2.88(2.01)$ \\
\hline \multicolumn{3}{|c|}{$\begin{array}{l}\text { PGY }=\text { postgraduate year; SD }=\text { standard deviation } \\
289 / 475(60.8 \%) \text { residents responded to the survey. Data are expressed either as means }\left( \pm \text { SD) and compared by } t \text {-tests }\left({ }^{*} P<0.01\right) \text { or as }\right. \\
\text { frequencies and compared by chi-square analysis }\left({ }^{\dagger} P<0.05\right) \text {. Percentages may not sum to } 100 \text { because of missing data }\end{array}$} \\
\hline
\end{tabular}




\section{Sleep Deprivation Impact (SDI) score}

The items comprising the SDI scale are listed in Table 2. Internal consistency of the 12-item SDI scale was 0.89 . Although both groups of residents agreed that individual performance was impaired by sleep deprivation, surgery residents scored significantly lower on the SDI scale (45.2 versus 51.5;
$P<0.01)$, implying they perceived sleep deprivation to have less impact on their performance. Group accounted for $15.4 \%$ of the variance in total SDI score.

When asked about procedural performance when deprived of sleep, surgery residents scored significantly lower than medicine residents (Table 3 ).

Table 2 Sleep Deprivation Impact (SDI) scores

After being Up All Night On Call, the next morning...
Surgery mean (SD)

$\begin{array}{lr}4.20(0.92)^{\star} & 4.49(0.79) \\ 3.55(1.34)^{*} & 4.08(1.04) \\ 3.76(1.04)^{\dagger} & 4.08(1.00) \\ 3.62(1.20)^{*} & 4.16(0.92) \\ 3.76(1.11)^{\star} & 4.54(0.78) \\ 3.67(1.03)^{*} & 4.32(0.80) \\ 4.07(.99)^{*} & 4.56(0.75) \\ 4.16(.89)^{*} & 4.51(0.84) \\ 3.70(1.13)^{*} & 4.18(0.91) \\ 2.93(1.28)^{*} & 3.75(1.09) \\ 4.14(.81)^{*} & 4.51(0.84) \\ 3.59(1.21)^{\dagger} & 3.95(1.14) \\ 45.2(8.74)^{*} & 51.5(5.97)\end{array}$

I am more irritable

I am less empathetic towards my patients

My ability to interact with colleagues is diminished

I am less effective at communicating with patients and families

My ability to concentrate during morning patient rounds is diminished

My ability to retain information on a new patient is diminished

My motivation to learn is diminished

My desire to teach medical students is diminished

I am less effective at formulating diagnoses or making clinical judgments

My ability to write appropriate discharge orders and prescriptions is diminished

It takes me longer to do things

I feel unsafe driving home

Total (SDI score)

\section{Non-surgery mean (SD)}

$\mathrm{SD}=$ standard deviation

Residents' responses to a series of 12 statements suggesting a detrimental effect of sleep deprivation on different aspects of performance. Mean scores of all items, in addition to the mean summed score, are reported ( ${ }^{*} P<0.01,{ }^{\dagger} P<0.05, t$-test)

Likert scale: $1=$ strongly disagree; $2=$ somewhat disagree; $3=$ no opinion; $4=$ somewhat agree; $5=$ strongly agree

Table 3 Impact of sleep deprivation on procedural skills

After being Up All Night On Call, the next morning..

Surgery mean (SD)

Medicine mean (SD)

I am less effective performing technical skills

My ability to learn new procedural skills is diminished

$3.56(1.09)^{*}$
$3.65(1.16)^{*}$
$3.08(1.31)^{\star}$
$2.73(1.20)$
$4.25(0.86)$

$4.17(1.06)$

$4.29(1.15)$

$3.77(1.27)$

NA

NA

My ability to perform a short ( 1 hour) OR case is diminished
My ability to perform a long (6 hour) OR case is diminished

SD = standard deviation; $N A=$ not applicable; $O R=$ operating room Residents' responses to a series of statements suggesting a detrimental effect of sleep deprivation on procedures. Mean Likert scores are reported $\left({ }^{*} P<0.01,{ }^{\dagger} P<0.05, t\right.$-test) Likert scale: 1 = strongly disagree; $2=$ somewhat disagree; $3=$ no opinion; $4=$ somewhat agree; $5=$ strongly agree ${ }^{\S}$ To manage a code blue; a resuscitative effort involving a pulseless or unresponsive patient. 


\section{Perceived required amounts of sleep}

When comparing themselves with others, surgery residents were more likely to believe that they required less sleep than residents in other programmes and that they had adapted to sleep deprivation since the start of residency (Table 4). There was, however, no difference across specialty in the number of hours of sleep that residents reported needing to function well (4.6 versus 4.9 hours).

\section{Working late post-call}

Residents were asked how often they stayed late at work post-call, defined arbitrarily as longer than
1 hour after the 'post-call home at noon' rule mandated for residents in Ontario. Surgery residents reported staying late more frequently than nonsurgery residents (Table 5), and perceived pressure to stay late post-call.

\section{Subgroup analyses}

Analysis of data within groups revealed few differences. Within the surgery group female residents reported working longer hours than male residents (90.8 versus 81.6 hours; $P<0.01$ ) but showed no difference in ESS scores as a result (12.6 and 12.9, respectively). Within the non-surgery group, female residents were less likely to perceive themselves as

Table 4 Perceptions of amount of sleep required

How much sleep do you need...

Compared with most residents in other programmes

Compared with most residents in your programme

Compared with your own staff supervisors

Compared with yourself at the start of your residency

What is the minimum amount of sleep on call you need

to perform clinical duties adequately the next morning?

What is the minimum amount of sleep on call you need

to perform clinical duties well the next morning?
Surgery mean (SD)

$2.54(1.02) *$

$3.00(0.64)$

$2.88(0.99)$

$2.78(1.04)^{\dagger}$

2.6 hours $(1.8)$

4.6 hours (1.8)
Non-surgery mean (SD)

$3.27(0.94)$

$3.13(0.80)$

$2.98(0.99)$

$3.09(1.10)$

3.3 hours $(4.8)$

4.9 hours $(1.7)$

SD = standard deviation

Residents' responses about their own sleep requirements. Mean Likert scores are reported $\left(* P<0.01,{ }^{\dagger} P<0.05 t\right.$-test)

Likert scale: 1 = much less; 2 = slightly less; 3 = same; 4 = slightly more; 5 = much more

Table 5 Post-call work patterns and perceived pressure

\begin{tabular}{|lll}
\hline How often do you... & Surgery mean (SD) & $\begin{array}{c}\text { Non-surgery } \\
\text { mean (SD) }\end{array}$ \\
\hline ... Stay late to work post-call? & $4.63(0.78)^{\star}$ & $3.29(1.13)$ \\
... feel looked down upon by staff for not working late post-call? & $3.55(1.30)^{\star}$ & $2.12(1.15)$ \\
... feel looked down upon by other residents for not working late post-call? & $3.17(1.46)^{\star}$ & $1.74(0.96)$ \\
... excuse yourself from work post-call because you are too tired? & $1.48(0.70)$ & $1.59(0.93)$
\end{tabular}

Residents' responses about their post-call work patterns and sources of perceived pressure to stay longer. Mean Likert scores are reported (* $P<0.01, t$-test)

Likert scale: $1=$ never; 2 = almost never; 3 = sometimes; 4 = often; 5 = very often 
able to adapt to less sleep with time compared with male residents $(3.29$ versus $2.91 ; P<0.05)$. In both groups, junior residents (PGY-2) reported working longer hours per week compared with more senior colleagues, but only in the non-surgery group were the results significant (69.7 versus 59.9 hours; $P<0.01)$. Corresponding with this result, these junior residents scored significantly higher on the ESS (12.9 versus 8.54 for senior residents within the non-surgery group; $P<0.01$ ). Within the surgery group, senior residents scored lower on the SDI compared with junior residents (44.5 versus 48.7 ; $P<0.05)$ indicating a perception of increased resilience to sleep deprivation in this more experienced group.

Finally, some differences reflective of specialty were noted within the groups. Medicine residents reported working longer hours per week (67.3 versus 50.6 hours; $P<0.1)$ and were sleepier $(9.9$ versus 7.6 on the ESS; $P<0.01)$ than psychiatry residents. However, no difference was noted in SDI scores between these 2 specialties (51.6 for medicine and 51.3 for psychiatry).

\section{DISCUSSION}

\section{Work hours and sleepiness}

As has been reported in other institutions, ${ }^{16}$ surgery residents who participated in this study appear to work longer hours than residents in other specialties. Our data suggest that at least some of these additional work hours may occur during the post-call period, as surgery residents report leaving the hospital later than other residents. It is not surprising then that, on a daily basis, surgery residents report higher levels of sleepiness. Indeed, their level of sleepiness as scored on the ESS (mean $=12.8$ ) is cause for concern as a score this high may be considered pathological: patients with obstructive sleep apnoea report a mean score of $11.7^{14}$ and numerous studies have found an association between this condition and increased risk to personal safety as a result of excessive sleepiness (particularly in motor vehicle collisions). ${ }^{17}$ Likewise, there is now increasing evidence that sleepdeprived resident doctors may be more prone to committing medical errors and experience a higher incidence of automobile collisions. ${ }^{4,18}$ Such observations are not beyond the purview of local policy makers. In 2003, for example, the New Jersey State legislature recognised the dangers of excessive sleepiness by ratifying Maggie's Law. ${ }^{19}$ Not restricted to medical personnel, this law makes driving following more than 24 hours of sleeplessness a criminal offence.

\section{Perception of the effects of sleep deprivation}

Despite this increased potential for adverse events among surgery residents, in our study this group appeared less likely to appreciate the potential impact of sleep deprivation on their own performance, reporting significantly lower SDI scores than non-surgery residents. According to Cohen, ${ }^{20}$ an eta-square value of 0.154 (suggesting that $15.4 \%$ of variance in total score could be accounted for by group) represents a moderate effect size of specialty as a factor in SDI score. Despite differences in sleepiness scores and reported work hours between medicine and psychiatry residents in the non-surgery group, no differences in SDI scores were detected, suggesting that this phenomenon may indeed be unique to surgery.

This study is not the first to report differences in the perceived impact of sleep deprivation. Sexton et al. ${ }^{13}$ found that the potential impact of fatigue was better appreciated by airline pilots than by medical personnel, and that among health care workers, surgeons were more likely than anaesthesiologists to deny such effects. Together with our data on residents, these findings suggest that surgeons may perceive themselves as more resilient than other specialists, which is consistent with longstanding beliefs about the 'culture of surgery'. ${ }^{21,22}$ The observation that senior surgery residents' SDI scores were significantly lower than those of junior surgery residents further suggests that the surgery environment itself may reinforce these beliefs.

These results do raise questions concerning the validity of self-reported work hours, as perception of relative immunity from sleep deprivation may promote a culture of non-compliance with work hour restrictions. This would support the suggestion for policing not only work hours, as is currently done in the USA, but also resident sleepiness (e.g. by an objective neurophysiological measure of sleepiness such as the psychomotor vigilance task ${ }^{23}$ ) in order to augment the data on work hours reported.

\section{Optimism bias}

One explanation for the results presented here is that surgeons may be exhibiting a type of 'optimism bias' in which negative events are seen as less likely to occur to the individual than the group. ${ }^{24}$ Such a misperception has been reported in the 
transportation industry among taxi and truck drivers, who acknowledge the potential detrimental impact of fatigue on the safety of other drivers, but not on their own. ${ }^{25}$ In the current study, surgery residents reported needing significantly less sleep than residents in other programmes, but when asked pointedly how many hours of sleep they required to perform their job, their answer was no different from that of non-surgery residents (both groups reported requiring approximately 3 hours to function adequately and 5 hours to function well).

Alternatively, the possibility remains that surgeons are a self-selected group who are able to outperform others when sleep-deprived. To date, however, no study has directly compared performance on clinically relevant tasks among different groups of doctors. On the contrary, recent well controlled studies have contributed to accumulating evidence that surgeons are indeed susceptible to the effects of sleep deprivation. ${ }^{2,26,27}$ With mounting evidence that doctor performance can be affected by sleep deprivation, it is important to promote an environment that acknowledges these potential risks to both patient and personal safety.

\section{Self-assessment}

Contributing to this optimism bias may be an inability to adequately judge performance when sleep-deprived. Dorrian $e t$ - $a .^{12}$ assessed night-shift workers on several standardised psychological tasks and concluded that subjects' ability to predict performance impairment was moderate at best. Similarly, poor to modest correlations have been reported between perceived and actual performance during sleepdeprived states in both simulated and on-road driving tasks. ${ }^{28,29}$ Interestingly, results from the study by Philip et $a l .{ }^{28}$ indicated that self-assessment was weakest in the on-road situation, where real-life factors may indeed motivate subjects to fight against fatigue but may also lead to an inflated assessment of actual performance. The increased pressure to stay late post-call reported in the current study by surgery residents as compared with non-surgery residents may provide just such a motivational factor in the clinical environment. Further research is needed to determine if there is any foundation for such a claim.

\section{Study limitations}

An anonymous survey design was chosen for this study as it was felt to be the best way of encouraging both participation and honest responses, given the sensitivity of some of the questions. Residents at only
1 academic institution were surveyed and the applicability to other training programmes or to staff doctors has not been established (although some degree of generalisability can be inferred, given that this study was carried out in 6 different teaching hospitals). Although we had a very high response rate for this type of study $(60.4 \%)$, it is possible that non-responders differed from the group of residents who responded to the survey. However, we were able to obtain information on the population of non-responders and found that they were very similar to the responders in age, gender distribution and PGY-level. In addition, the SDI scale consists of items that are worded in the negative voice, which may have prompted respondents. However, this was true for all 3 groups of residents and it is the comparison of responses that is most interesting and relevant in this study. Clearly, as this is a novel instrument, ongoing investigation of its reliability and validity are required.

Finally, the current study did not attempt to correlate perceived resident performance with actual clinical performance. Further work in this field should explicitly explore whether surgery residents possess an elevated resilience to the effects of sleep deprivation.

\section{CONCLUSIONS}

The results of the current study have important implications for both medical education and the practice of medicine. More specifically, they are consistent with the presence of an underlying culture within the surgery environment that is different from that in other specialties, and which may involve less willingness to accept the natural limitations of human performance. The existence of such a culture may explain, in part, the resistance of surgeons to recent work hour changes. In the USA, for example, although threats of disciplinary action against training programmes ensure high compliance rates to work hour restrictions, prominent opposition to such measures has originated almost exclusively from the profession of surgery. ${ }^{30,31}$ In Canada, where post-call work hour enforcement is less stringent, adherence to these guidelines is even more dependent on self-regulation. Optimism bias - such as has been described here - particularly if it is supported by the broader surgery culture, may promote non-compliance and ultimately endanger patient safety. ${ }^{24}$ Moreover, it seems likely that attitudes and behaviours learned during residency will continue into independent practice where no work hour restrictions 
currently exist. At a time when evidence for the negative effects of sleep deprivation is mounting, it is imperative that all doctors, as self-regulated professionals, have an accurate understanding of this important issue.

Contributors: SIW contributed to the study conception and design, acquisition, analysis and interpretation of data, and the drafting of the manuscript. JP contributed to the study conception and design, and acquisition, analysis and interpretation of data. BJM contributed to the study conception and design, and analysis and interpretation of data. CW contributed to the acquisition of data. MB and RKR contributed to the study conception and design. SJH contributed to the analysis and interpretation of data, and the drafting of the article. All authors contributed to the revision of the manuscript and gave it final approval. Acknowledgements: the authors acknowledge Dr Glenn Regehr and Chloe MacDonald of the University of Toronto. Funding: SIW and JP were supported by the Royal College of Physicians and Surgeons of Canada's Research Fellowship in Medical Education.

Conflicts of interest: none.

Ethical approval: the study was approved by the University of Toronto Research Ethics Board.

\section{REFERENCES}

1 Pilcher JJ, Huffcutt AI. Effects of sleep deprivation on performance: a meta-analysis. Sleep 1996;19 (4):31826.

2 Grantcharov TP, Bardram L, Funch-Jensen P, Rosenberg J. Laparoscopic performance after one night on call in a surgical department: prospective study. BMJ 2001;323 (7323):1222-3.

3 Landrigan CP, Rothschild JM, Cronin JW et al. Effect of reducing interns' work hours on serious medical errors in intensive care units. N Engl J Med 2004;351 (18):1838-48.

4 Arnedt JT, Owens J, Crouch M, Stahl J, Carskadon MA. Neurobehavioural performance of residents after heavy night call vs after alcohol ingestion. JAMA 2005;294 (9):1025-33.

5 Ferrara M, De Gennaro L. How much sleep do we need? Sleep Med Rev 2001;5 (2):155-79.

6 Bonnet MH, Arand DL. We are chronically sleep deprived. Sleep 1995;18 (10):908-11.

7 Papp KK, Stoller EP, Sage P, Aikens JE, Owens J, Avidan A, Phillips B, Rosen R, Strohl KP. The effects of sleep loss and fatigue on resident physicians: a multi-institutional, mixed-method study. Acad Med 2004;79 (5):394406.

8 Rosen IM, Bellini LM, Shea JA. Sleep behaviors and attitudes among internal medicine housestaff in a U.S. university-based residency program. Acad Med 2004;79 (5):407-16.
9 Whang EE, Perez A, Ito H, Mello MM, Ashley SW, Zinner MJ. Work hours reform: perceptions and desires of contemporary surgical residents. J Am Coll Surg 2003;197 (4):624-30.

10 Rosen IM, Gimotty PA, Shea JA, Bellini LM. Evolution of sleep quantity, sleep deprivation, mood disturbances, empathy, and burnout among interns. Acad Med 2006;81 (1):82-5.

11 Eva KW, Regehr G. Self-assessment in the health professions: a reformulation and research agenda. Acad Med 2005;10 (Suppl):46-54.

12 Dorrian J, Lamond N, Holmes AL, Burgess HJ, Roach GD, Fletcher A, Dawson D. The ability to self-monitor performance during a week of simulated night shifts. Sleep 2003;26 (7):871-7.

13 Sexton JB, Thomas EJ, Helmreich RL. Error, stress, and teamwork in medicine and aviation: cross-sectional surveys. BMJ 2000;320 (7237):745-9.

14 Johns MW. A new method for measuring daytime sleepiness: the Epworth Sleepiness Scale. Sleep 1991;14 (6):540-5.

15 Chervin RD, Aldrich MS, Pickett R, Guilleminault C. Comparison of the results of the Epworth Sleepiness Scale and the Multiple Sleep Latency Test. J Psychosom Res 1997;42 (2):145-55.

16 Brotherton SE, Simon FA, Etzel SI. US graduate medical education, 2000-2001. JAMA 2001;286 (9): 1056-60.

17 Sassani A, Findley LJ, Kryger M, Goldlust E, George C, Davidson TM. Reducing motor vehicle collisions, costs, and fatalities by treating obstructive sleep apnoea syndrome. Sleep 2004;27 (3):453-8.

18 Barger LK, Cade BE, Ayas NT, Cronin JW, Rosner B, Speizer FE, Czeisler CA. Harvard Work Hours, Health, and Safety Group. Extended work shifts and the risk of motor vehicle crashes among interns. $N$ Engl J Med 2005;352 (2):125-34.

19 An Act Concerning Vehicular Homicide and Amending N.J.S.2C: 11-5 (New Jersey Chapter Law PL03, c.143, 5 August 2003). http://www.njleg.state.nj.us/2002/ Bills/PL03/143_HTM. (Accessed 27 November 2007.)

20 Cohen J. Statistical Power for the Behavioral Sciences., 2nd edn. Hillsdale, NJ: Lawrence Erlbaum Associates 1988.

21 Whang EE, Mello MM, Ashley SW, Zinner MJ. Implementing resident work hour limitations: lessons from the New York State experience. Ann Surg 2003;237 (4):449-55.

22 Cassell J. Expected Miracles: Surgeons at Work. Philadelphia, PA: Temple University Press 1991.

23 Jewett ME, Dijk DJ, Kronauer RE, Dinges DF. Dose-response relationship between sleep duration and human psychomotor vigilance and subjective alertness. Sleep 1999;22 (2):171-9.

24 Weinstein ND. Optimistic biases about personal risks. Science 1989;246 (4935):1232-3.

25 Dalziel JR, Job RF. Motor vehicle accidents, fatigue and optimism bias in taxi drivers. Accid Anal Prev 1997;29 (4):489-94. 
26 Taffinder NJ, McManus IC, Gul Y, Russell RC, Darzi A. Effect of sleep deprivation on surgeons' dexterity on laparoscopy simulator. Lancet 1998;352 (9135):1191.

27 Eastridge BJ, Hamilton EC, O'Keefe GE, Rege RV, Valentine RJ, Jones DJ, Tesfay S, Thal ER. Effect of sleep deprivation on the performance of simulated laparoscopic surgical skill. Am J Surg 2003;186 (2):169-74.

28 Philip P, Sagaspe P, Taillard J, Moore N, Guilleminault C, Sanchez-Ortuno M, Akerstedt T, Bioulac B. Fatigue, sleep restriction, and performance in automobile drivers: a controlled study in a natural environment. Sleep 2003;26 (3):277-80.

29 Arnedt JT, Wilde GJ, Munt PW, MacLean AW. Simulated driving performance following prolonged wakefulness and alcohol consumption: separate and combined contributions to impairment. J Sleep Res 2000;9 (3):233-41.

30 Browne BJ, Madden RL, Kurbanov A, Lipkowitz GS. Effects of limited work hours on surgical training. J Am Coll Surg 2003;196 (4):661.

31 Fischer JE. Continuity of care: a casualty of the 80-hour work week. Acad Med 2004;79 (5):381-3.

Received 14 July 2006; editorial comments to authors 16 November 2006, 16 April 2007; accepted for publication 22 August 2007 